\title{
Vector solitons in harmonic mode-locked erbium-doped fiber lasers
}

\author{
Tatiana Habruseva $^{* a}$, Mkhitar Mkhitaryan $^{\mathrm{b}}$, Chengbo Mou ${ }^{\mathrm{a}}$, Aleksey Rozhin ${ }^{\mathrm{a}}$, Sergei K. Turitsyn ${ }^{\mathrm{a}}$, \\ and Sergey V. Sergeyev ${ }^{\mathrm{a}}$ \\ ${ }^{a}$ Aston Institute of Photonic Technologies, Aston University, Birmingham, B4 7ET, UK; \\ ${ }^{b}$ Department of General and Applied Physics, Moscow Institute of Physics and Technology, \\ Dolgoprudny, Russia
}

\begin{abstract}
We report experimental study of vector solitons for the fundamental and harmonic mode-locked operation in erbiumdoper fiber lasers with carbon nanotubes based saturable absorbers and anomalous dispersion cavities. We measure evolution of the output pulses polarization and demonstrate vector solitons with various polarization attractors, including locked polarization, periodic polarization switching, and polarization precession.
\end{abstract}

Keywords: Mode locked lasers, solitons, polarization dynamics, fiber lasers

\section{INTRODUCTION}

To describe a train of short pulses with a specific shape defined by a complex interplay and balance between the effects of gain/loss, dispersion, and nonlinearity in mode-locked fiber lasers (MLFLs), the concept of dissipative solitons has been recently introduced ${ }^{1,2}$. With an increased gain in active medium, fundamental soliton becomes unstable and more complex waveforms can appear, viz. multi-pulsing ${ }^{3,4}$, harmonic mode locking ${ }^{5}$, soliton molecules ${ }^{1,2,6-13}$, and soliton rain $^{1,14}$. The multi-pulsing and harmonic mode-locking arises as a result of an interplay between the laser cavity bandwidth constraints and the energy quantization associated with the resulting mode-locked pulses ${ }^{3,4}$.

Harmonic mode-locking provides the technique for high repetition rates pulse generation in passively MLFLs, which is of great interest for telecommunications and sensing applications. Typically MLFLs operate at repetition rates in the order of $\mathrm{MHz}$ due to relatively long laser cavities, however, the increase of the pump power results in solitons breakup leading to multi-pulsing and harmonic mode-locking. When compared to active mode-locking techniques, passive modelocking has reduced complexity and a potential to obtain shorter optical pulses at high repetition rates. Polarization dynamics in MLFLs is important to reveal the pulse train stability, which is vital for fibre optic communications and alloptical cock recovery.

Recently, the vector nature of dissipative solitons has been exploited to reveal polarization dynamics of dissipative solitons or so-called vector dissipative solitons (VDSs) ${ }^{4,8,13,15}$. Apart from the fundamental interest, VDSs provide a base for advances in increased capacity in fiber optic telecommunications, secure communications, trapping and manipulation of atoms and nanoparticles, control of magnetization in data storage devices and many other areas ${ }^{16-25}$. In our previous publications we have already studied experimentally conditions for emerging and suppression of VDSs for multi-pulse and bound-states operation ${ }^{4,8,13,15}$.

In this paper, we address issues related to the noise properties of harmonic mode-locked operation and control of state of polarization (SOPs) in both fundamental and harmonic mode-locked regime. Harmonic MLFLs based on carbon nanotubes (CNTs) demonstrated sub-picosecond pulses at high repetition rates ${ }^{26}$.We present experimental study of polarization attractors of fundamental and harmonic mode-locked regimes for two fiber lasers with different gain fibers. Unlike polarization dynamics of vector solitons on the Poincare sphere demonstrated by Akhmediev, Soto-Crespo ${ }^{27}$ and Komarov et al. ${ }^{28}$, we demonstrate new insight related to contribution of polarization hole burning in an active medium to the polarization dynamics.

\section{EXPERIMENTAL SET-UP AND RESULTS}

We used two fiber laser configurations in these studies. The laser 1 ring cavity shown in Fig. 1 with a total cavity length of $8 \mathrm{~m}$ (a) included high concentration erbium doped fiber (2m of LIEKKI ${ }^{\mathrm{TM}}$ Er80-8/125), single mode fiber (SMF-28)

Nonlinear Optics and Its Applications VIII; and Quantum Optics III, edited by Benjamin J. Eggleton, Alexander L. Gaeta, Neil G. R. Broderick, Alexander V. Sergienko, Arno Rauschenbeutel, Thomas Durt, Proc. of SPIE Vol. 9136, 91360X - (c) 2014 SPIE · CCC code: 0277-786X/14/\$18 · doi: 10.1117/12.2052445 
with anomalous dispersion, a 99/1 tap, an optical isolator, a polarization controller (PC), a wavelength division multiplexing (WDM) coupler, an output coupler, and a fast (370 fs relaxation time) saturable absorber based on carbon nanotubes (CNTs). The laser 2 configuration was similar to that of the laser 1, but with different erbium doped fiber used as a gain: $40 \mathrm{~cm}$ of LIEKKI ${ }^{\mathrm{TM}} \operatorname{Er110}-4 / 125$. In both cases, the gain fiber was pumped at $976 \mathrm{~nm}$ by a laser diode via a 980/1550 nm WDM coupler and a PC. The maximum pump laser diode current was about $355 \mathrm{~mA}$, which corresponded to $170 \mathrm{~mW}$ of optical power. We used a single-wall CNTs polymer film embedded between standard FC/APC fiber connectors as a saturable absorber. CNTs films preparation and characterization was as described in Ref. 28. In experiments, the pump current of the laser diode was increased and various polarization attractors were obtained by varying both the in-cavity and pump PCs for a given pump power.

The laser outputs were characterized using optical and electronic spectrum analyzers (ANDO AQ6317B and HP8562), an inline polarimeter (Thorlabs, IPM5300) and a fast oscilloscope (Tektronix DPO7254). The dynamics of SOP was characterized using the inline polarimeter, which measured the normalized Stokes parameters $s_{1}, s_{2}$, $s_{3}$, output power and degree of polarization (DOP) producing 1024 samples with $1 \mu$ s resolution ( 25 round-trip times).

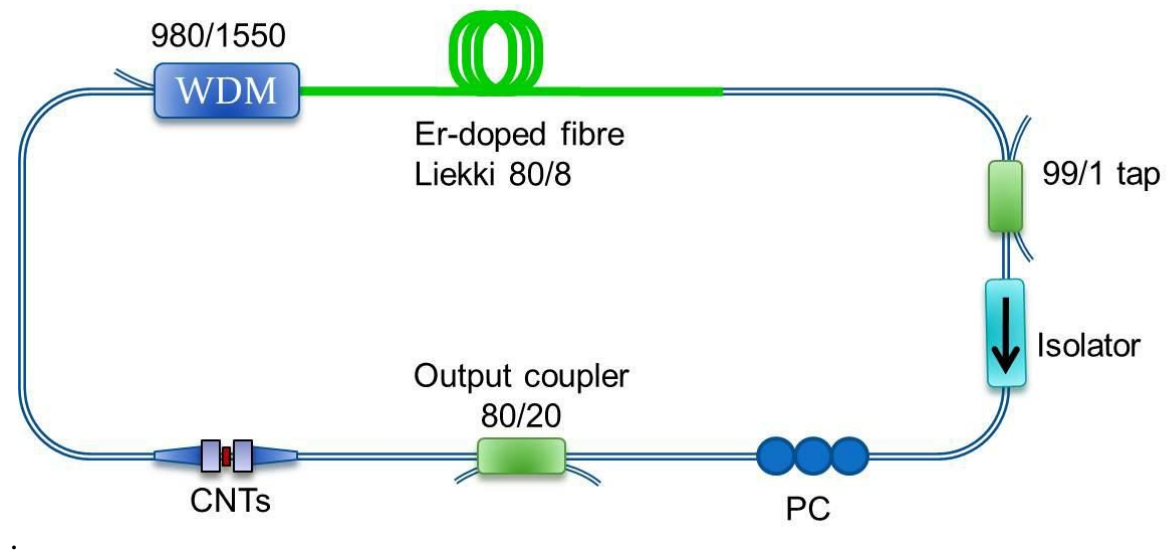

Figure 1. Configuration of Erbium-doped fiber laser with single wall carbon nanotubes (CNTs) saturable absorber. PC -polarization controller, WDM -- wavelength division multiplexing coupler, CNTs - carbon nanotubes based saturable absorber.

The Stokes parameters are related to the output powers of two linearly cross- polarized SOPs $|u|^{2}$ and $|v|^{2}$, and phase difference between them $\Delta \phi$ as follows:

$$
S_{0}=|u|^{2}+|v|^{2}, S_{1}=|u|^{2}-|v|^{2}, S_{2}=2\left|u\left\|v\left|\cos \Delta \phi, S_{3}=2\right| u\right\| v\right| \sin \Delta \phi
$$

The normalized Stokes parameters $s 1, s 2, s 3$, and a degree of polarization (DOP) are defined as:

$$
s_{i}=\frac{S_{i}}{\sqrt{S_{1}^{2}+S_{2}^{2}+S_{3}^{2}}}, \quad D O P=\frac{\sqrt{S_{1}^{2}+S_{2}^{2}+S_{3}^{2}}}{S_{0}},(i=1,2,3)
$$

The evolution of normalized Stokes parameters over time produces a track on the Poincarè sphere, which is commonly used to visualize polarization dynamics. In experiments, the pump power was varied and various polarization attractors were obtained by tuning both the intra-cavity and pump laser PCs.

Pump current was tuned between 50 and to $400 \mathrm{~mA}$, and various harmonics were achieved in the laser operation. Pump power increase resulted in the multiple-pulsing and harmonic mode-locking, with a higher pump power leading to a larger number of solitons and higher harmonics. This multi-pulsing arises due to limited cavity bandwidth constraints ${ }^{3,5}$. Soliton spectral bandwidth increase with the pump power is limited by the cavity gain bandwidth, which forces a single pulse split into two pulses with energy divided between them and spectral bandwidth within the gain 
bandwidth window. Low noise harmonic mode-locking was achieved with over $50 \mathrm{~dB}$ sidebands suppression ratio up to 10th harmonic. The sidebands increased with harmonic number, as shown in Figure 2. We achieved up to $13^{\text {th }}$ harmonic for both laser 1 and laser 2.

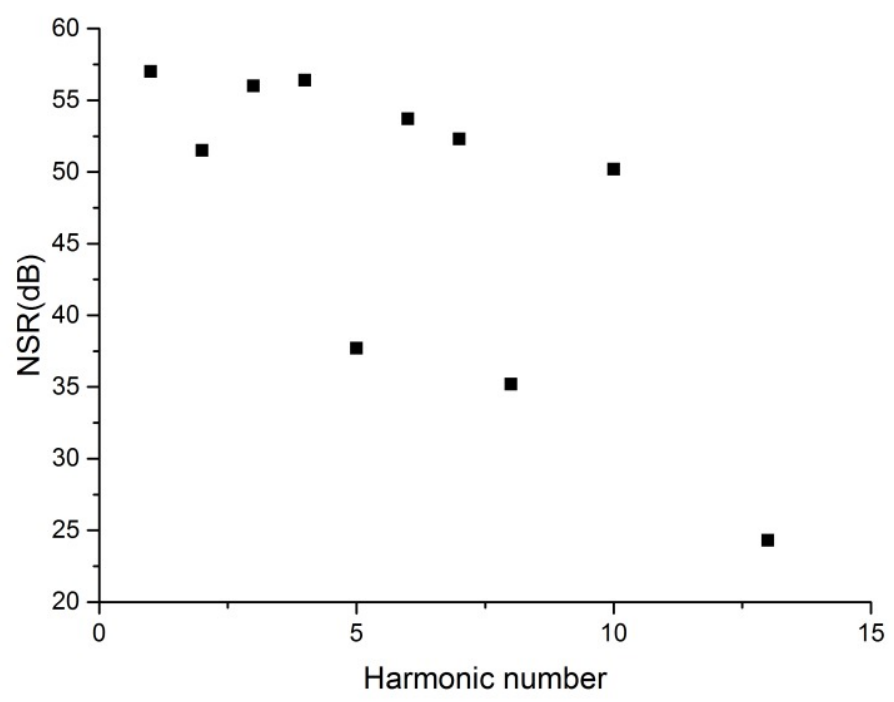

Figure 2. Sidebands suppression ratio versus harmonic number. Laser 1.

\section{POLARIZATION ATTRACTORS}

Tuning of the both pump and intra-cavity PCs lead to a large diversity of the laser operation regimes, including harmonic mode-locking, bound states and multi-pulsing. By varying the intra-cavity PC we could achieve VDSs with
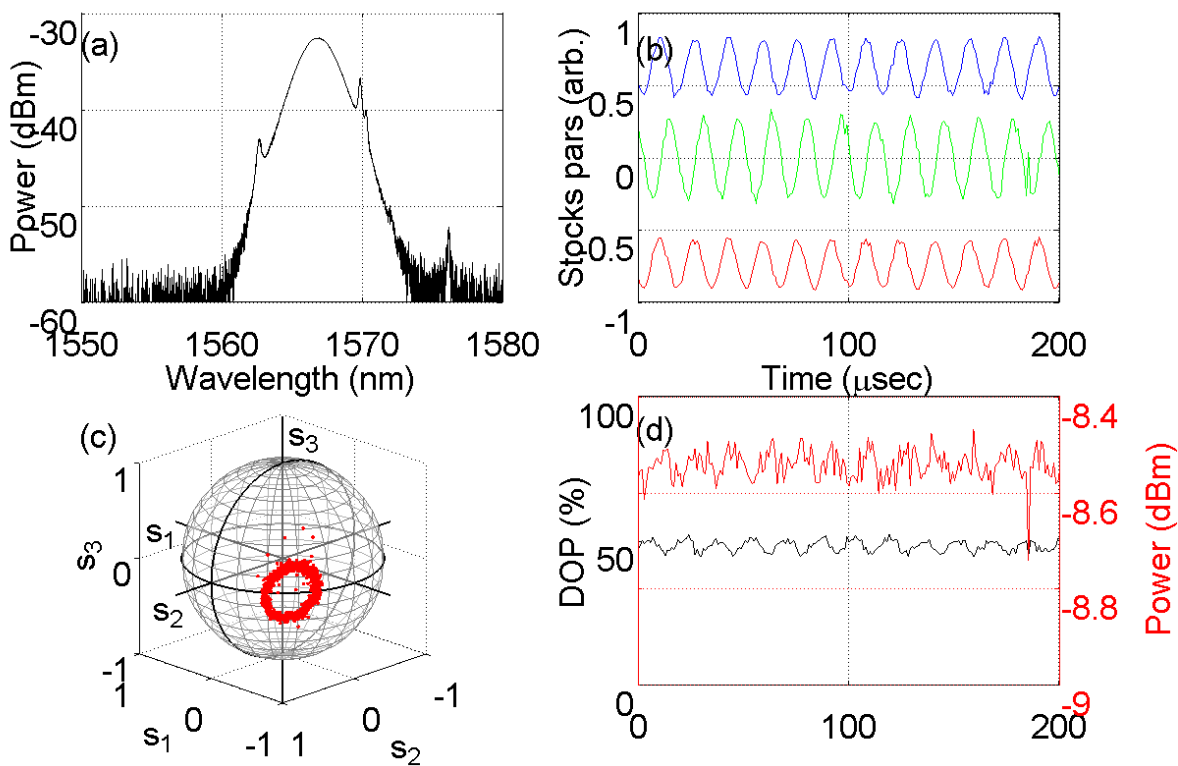

Figure 3. VDSs with circularly evolving SOP. (a) optical spectrum, (b) normalized Stokes parameters $\mathrm{s}_{1}$ (red), $\mathrm{s}_{2}$ (blue), $\mathrm{s}_{3}$ (green), (c) Poincarè sphere, (d) degree of polarization (black) and output power (red). Pump LD current $150 \mathrm{~mA}$. 
different polarization dynamics, such as polarization-locked vector solitons, solitons with precessing, switching and chaotic SOP ${ }^{4}$. Polarization attractors measured for fundamental mode-locking for laser 1 are shown in Figs. 3-4. Figure 3 (a) demonstrates a typical spectrum of a soliton operation with Kelly sidebands, the oscilloscope traces and electronic spectrum revealed fundamental mode-locking operation.

Figure 3 (c) demonstrates slow circularly evolving SOP evolution. Similar polarization dynamics was also observed for the second harmonic and multi-pulse operation. The evolution of the Stokes parameters shows periodic dynamics of SOP with a period of $\sim 17 \mu \mathrm{s}$. This exotic behavior is described in the next discussion section. Figure 4 shows VDSs at the fundamental harmonic with the sector-type attractor with grouping at two orthogonal SOPs (Fig.4 (c)).
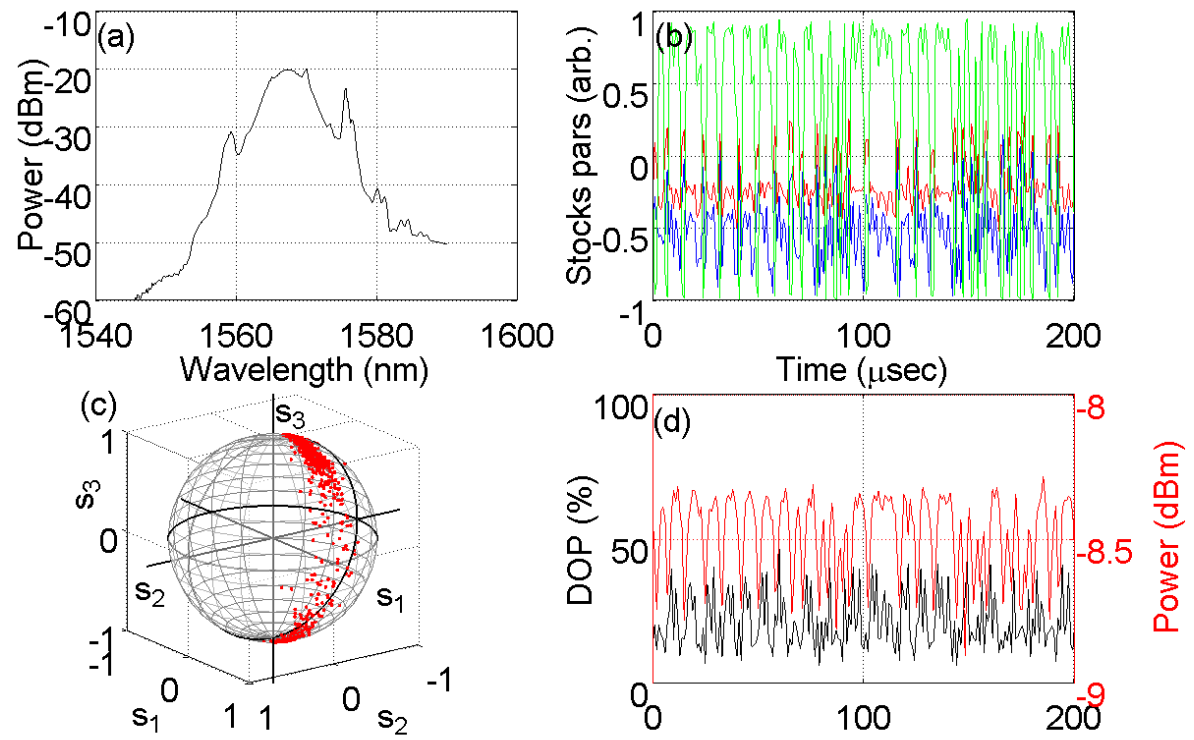

Figure 4. VDSs with conical polarization attractor. (a) optical spectrum, (b) normalized Stokes parameters $s_{1}$ (red), $s_{2}$ (blue), $s_{3}$ (green), (c) Poincarè sphere, (d) degree of polarization (black) and output power (red).
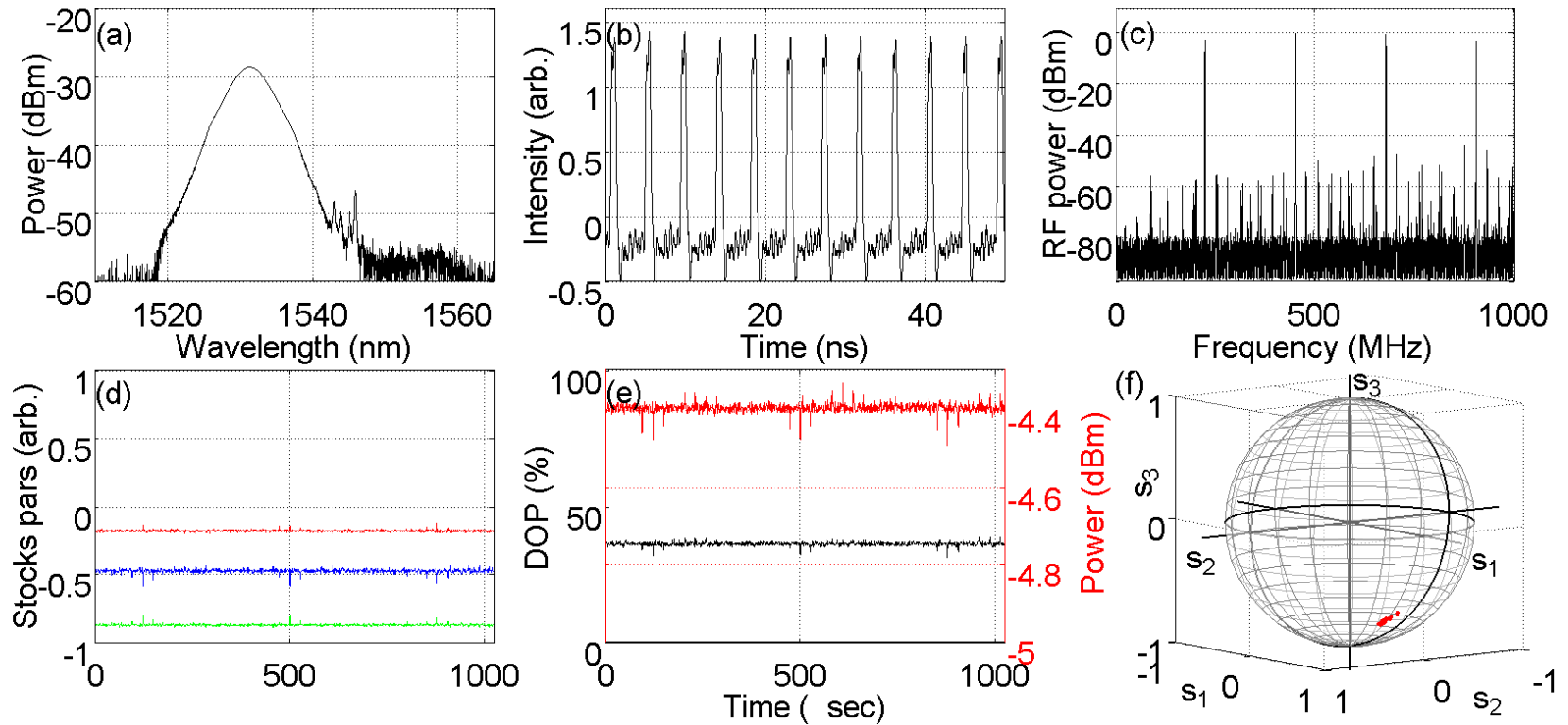

Figure 5. VDSs at the $8^{\text {th }}$ harmonic with locked polarization. (a) optical spectrum, (b) pulse train, (c) electronic spectrum, (d) normalized Stokes parameters, (e) degree of polarization (black) and output power (red), (f) Poincarè sphere. 
Polarization attractors measured for laser 2 are shown in Figs. 5-7. Figure 5 shows polarization-locked VDSs at $8^{\text {th }}$ harmonics. Polarization-locked VDSs could be achieved for different harmonics studied and fundamental mode-locking operation as well. Figures 6 and 7 show VSs at $11^{\text {th }}$ harmonic with localized (Fig. 6) and chaotic (Fig. 7) polarization. Polarization-locked VSs were also achieved at $11^{\text {th }}$ harmonic. Remarkably, optical (a), RF spectra (c) and pulse trains (b) were nearly identical for all these regimes, and only polarization attractors could reveal the differences.
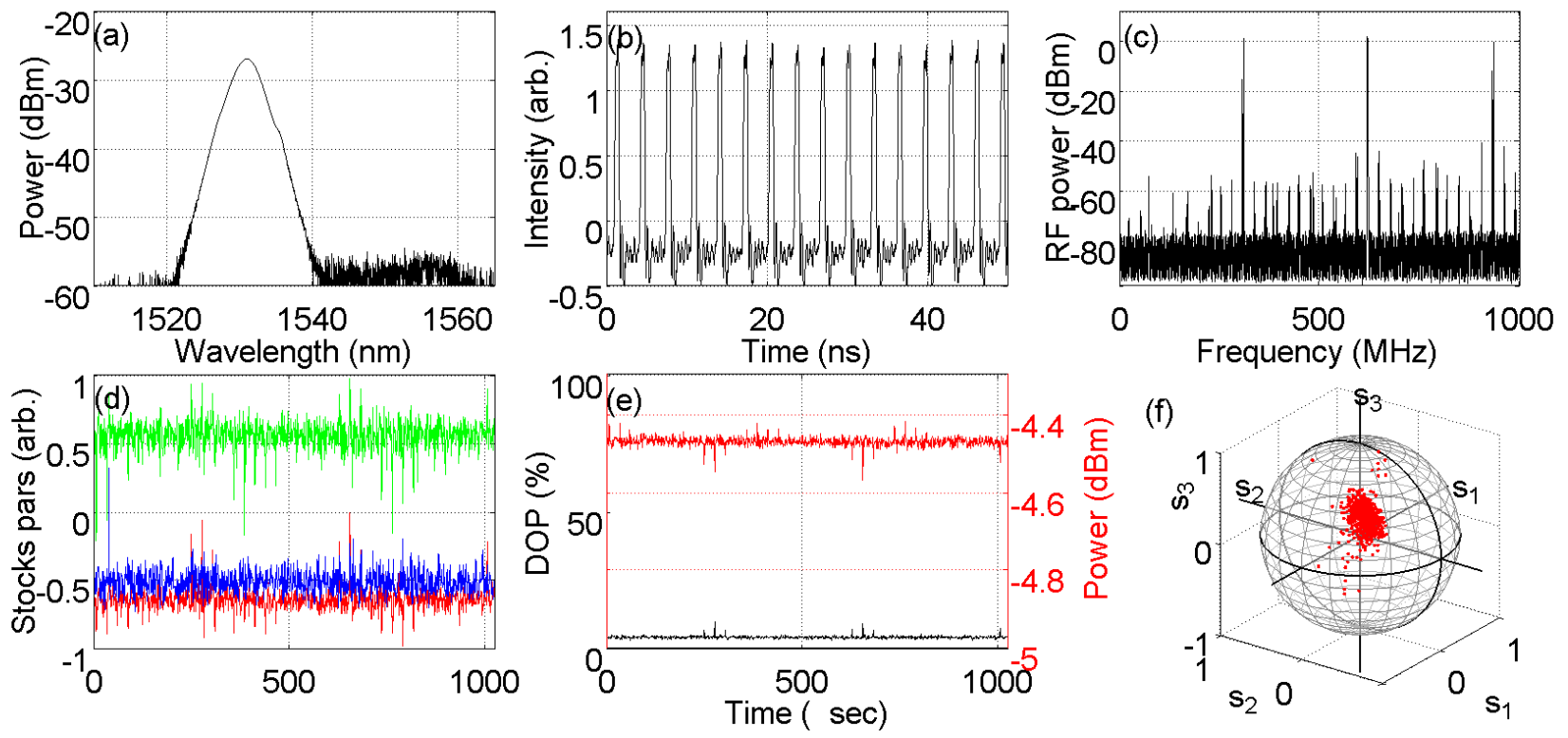

Figure 6. VDSs at the $11^{\text {th }}$ harmonic with polarization localized in a disk area. (a) optical spectrum, (b) pulse train, (c) electronic spectrum, (d) normalized Stokes parameters, (e) degree of polarization (black) and output power (red), (f) Poincarè sphere.
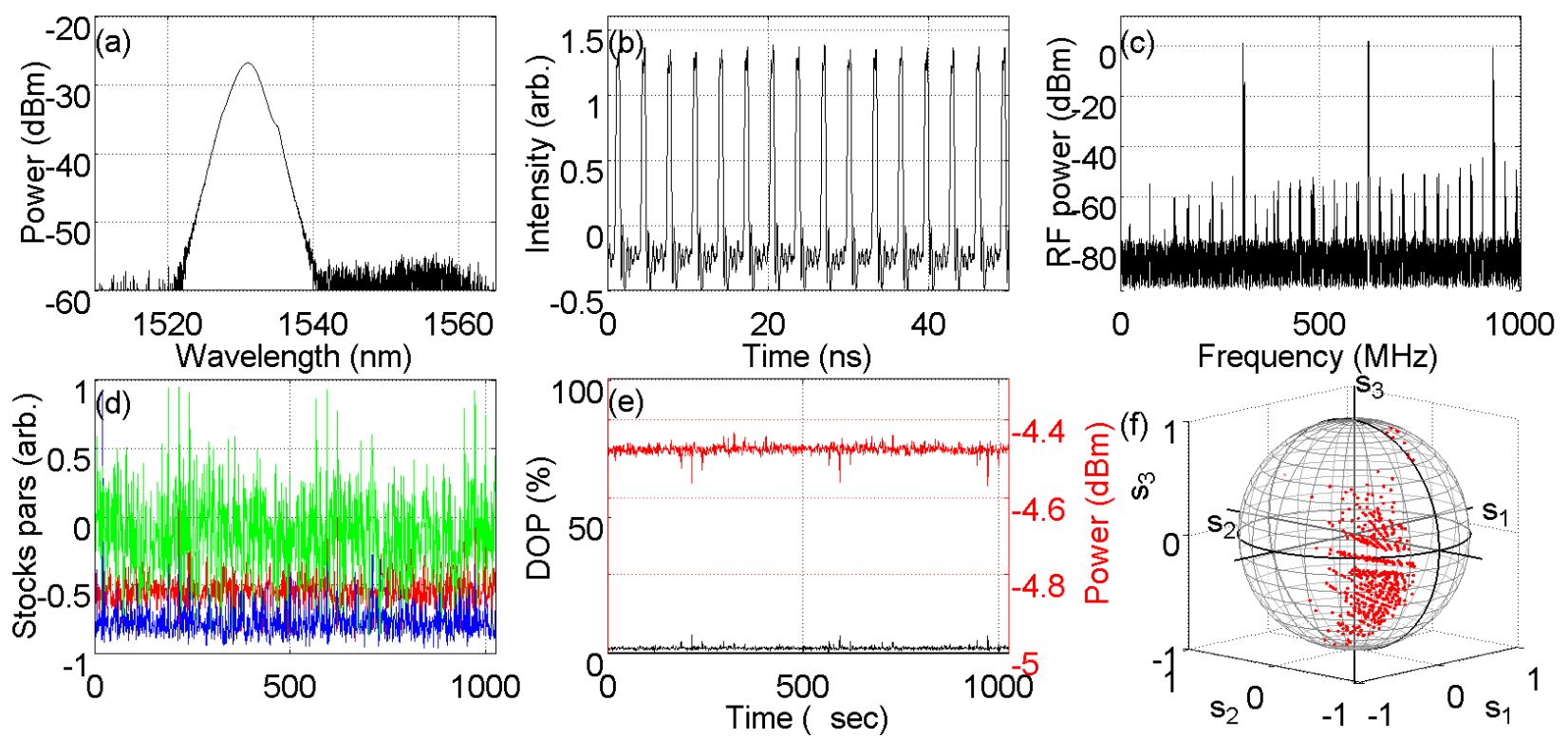

Figure 7. VDSs at the $11^{\text {th }}$ harmonic with chaotic polarization. (a) optical spectrum, (b) pulse train, (c) electronic spectrum, (d) normalized Stokes parameters, (e) degree of polarization (black) and output power (red), (f) Poincarè sphere. 


\section{DISCUSSION}

As shown by Akhmediev and Soto-Crespo ${ }^{27}$ and Komarov et al ${ }^{28}$, the eigenstates in a fiber in the presence of linear birefringence and circular birefringence caused by nonlinear self-phase modulation are split into two pairs:

$$
S^{(1,2)}=\left(\begin{array}{c}
S_{0} \\
\pm S_{0} \\
0 \\
0
\end{array}\right), \quad S^{(3,4)}=\left(\begin{array}{c}
S_{0} \\
-\alpha \\
0 \\
\pm \sqrt{S_{0}^{2}-\alpha^{2}}
\end{array}\right)
$$

Here $\mathrm{S}_{0}=$ const and $\alpha$ is determined by the ratio of linear to circular birefringence strength. In mode locked laser, incavity polarization controller contributes both into linear and circular birefringence, whereas anisotropy induced by pump light is suppressed due to relaxation orientation caused by excitation migration ${ }^{29}$. Mode-locked operation also changes the birefringence in the cavity (both linear and circular) due to polarization hole burning and so it leads to pulseto-pulse evolution of the SOP. If the beat length for the anisotropy caused by in-cavity polarization controller and polarization hole burning is equal to the round trip and pulse-to-pulse power is constant, then pulse SOP is not changed and so vector soliton is polarization locked ${ }^{13}$. In the case of round trip distance being equal to half or third of beat length, the SOP is reproduced in two or three round trips and, therefore, polarization switching takes the forms shown in Fig. 4. With increased pump power, amplitude instability emerges and leads to periodical oscillations of the output power. If the round trip is equal to the rational part of the beat length then SOP after few round trips will slightly deviate from the initial one and can only reproduce itself for the period of pulse power oscillations. Otherwise, pulse-to-pulse SOPs cannot be reproduced and so the SOPs fill densely the circle on Poincare sphere, as shown in Fig. 3 (c).

\section{CONCLUSIONS}

We have revealed new types of vector solitons in a carbon nanotube mode locked fiber laser with anomalous dispersion cavity. The observed evolution of the states of polarization includes periodic polarization switching between two SOPs, and circular evolution of SOP. We achieved high harmonics operation with sideband suppression ratio of over $50 \mathrm{~dB}$ up to $10^{\text {th }}$ harmonic. Our experimental studies can help to design new types of lasers with controlled dynamical states of polarization that are of a special high interest for applications in fiber optic telecommunications ${ }^{22-25}$, in nano-optics (trapping and manipulation of nanoparticle and atoms ${ }^{19}$ ), and spintronics (vector control of magnetization ${ }^{20}$ ).

\section{ACKNOWLEDGMENTS}

This work was funded by the Marie Curie Action FP7-PEOPLE-2011-IEF, HARMOFIRE project, Grant No 299288, by the EPSRC (project UNLOC, EP/J017582/1), European Research Council (ULTRALASER) and FP7-PEOPLE-2012 IAPP (project GRIFFON, No 324391).

\section{REFERENCES}

[1] Akhmediev, N., and Ankiewicz, A. Solitons, "Nonlinear Pulses and Beams". Chapman and Hall, London (1979).

[2] Grelu, Ph., and Akhmediev, N. "Dissipative solitons for mode-locked lasers. Nature Photon. 26, 84-92 (2012).

[3] Li, F., Wai, P. K. A., and Kutz, J. N. "Geometrical description of the onset of multipulsing in mode-locked laser cavities". J. Opt. Soc. Am. B 27, 2068-2077 (2010).

[4] Sergeyev, S., Mou, Ch., Rozhin, A., and Turitsyn, S. K."Vector Solitons with Locked and Precessing States of Polarization", Opt. Exp., 20, 27434-27440 (2012).

[5] Grudinin, B., and Gray, S. Passive harmonic mode locking in soliton fiber lasers. J. Opt. Soc. Am. B 14, 144154 (1997).

[6] Malomed B. A., Bound-States of Envelope Solitons, Phys. Rev. E 47, 2874-2880 (1993). 
[7] Grelu, Ph., Belhache, F., Gutty, F., and Soto-Crespo, J. M. "Phase-locked soliton pairs in a stretched-pulse fiber laser". Opt. Lett. 27, 966-968 (2002).

[8] Mou, Ch., Sergeyev, S. V., Rozhin, A., and Turitsyn, S. K, "Bound state vector solitons with locked and precessing states of polarization", Opt. Exp., 21, 26868-26875 (2013).

[9] Ortac, B., Zavyalov, A., Nielsen, C. K., Egorov, O., Iliew R., Limpert, J., Lederer, F., and Tunnermann, A. "Observation of soliton molecules with independently evolving phase in a mode-locked fiber laser". Opt. Lett. $35,1578-1580$ (2010).

[10] Wu, X., Tang, D. Y., Luan, X. N., and Zhang, Q. "Bound states of solitons in a fiber laser mode locked with carbon nanotube saturable absorber". Opt. Commun. 284, 3615-3618 (2011).

[11]Li, X. L., Zhang, S. M., Meng, Y. C., Hao, Y. P., Li, H. F., Du, J., and Yang, Z. J. "Observation of soliton bound states in a graphene mode locked erbium-doped fiber laser". Laser Physics 22, 774-777 (2012).

[12] Gui, L. L., Xiao, X. S., and Yang, C. X. "Observation of various bound solitons in a carbon-nanotube-based erbium fiber laser". JOSA B 30, 158-164 (2013).

[13] Tsatourian, V., Sergeyev, S. V., Mou, Ch., Rozhin, A., Mikhailov, V., Westbrook, P., Rabin, B., and Turitsyn, S.K. "Polarisation Dynamics of Vector Soliton Molecules in Mode Locked Fibre Laser", Scientific Reports, $3: 3154$, DOI: 10.1038/srep03154 (2013).

[14] Chouli, S., and Grelu, Ph. "Soliton rains in a fiber laser: An experimental study". Phys. Rev. A 81, 063829 (2010).

[15] Sergeyev, S.V., Mou, Ch., Turitsyna, E. G., Rozhin, A., Turitsyn, S.K., and Blow, K. "Spiral Attractor Created by Vector Solitons", Light: Science \& Applications, 3:e131, DOI:10.1038/lsa.2014.12 (2014).

[16] Udem, Th., Holzwarth, R., and Hänsch, T.W. "Optical frequency metrology". Nature 416, 233-237 (2002).

[17] Mandon, J., Guelachvili, G., Picqué, N. "Fourier transform spectroscopy with a laser frequency comb". Nature Photon 25, 99-102 (2009).

[18] Hillerkuss, D., Schmogrow, R., Schellinger, T., Jordan, M., Winter, M., $\quad$ Huber, G.,T. et al. "26 Tbit s 121 line- rate super- channel transmission utilizing all-optical fast Fourier transform processing". Nature Photon. 5, 364-371 (2011).

[19] Jiang ,Y., Narushima, T., Okamoto, H. "Nonlinear optical effects in trapping nanoparticles with femtosecond pulses". Nature Phys. 6, 1005-1009 (2010).

[20] Kanda, N., Higuchi, T., Shimizu, H., Konishi, K.,Yoshioka , K., and Kuwata-Gonokami, M. "The vectorial control of magnetization by light”. Nature Comm. 2:362 doi, 10.1038/ncoms1366 (201).

[21] Van Wiggeren, G.D., and Roy, R. "Communication with Dynamically Fluctuating States of Light Polarization". Phys. Rev. Lett. 88, 097903 (2002).

[22] Arahira, S., Takahashi, H., Nakamura, K., Yaegashi, H., and Ogawa, Y. "Polarization-, wavelength-, and filter free all-optical clock recovery in a passively mode-locked laser diode with orthogonally pumped polarization diversity configuration," IEEE J. Quantum Electron. 45, 476 -487 (2009).

[23] Kuri, T., Toda, H., Olmos, J., and Kitayama, K. "Reconfigurable dense wavelength-division-multiplexing millimeter-waveband radio-over-fiber access system technologies," J. Lightwave Technol. 28, 2247-2257 (2010).

[24] Serena, P., Rossi, N., Bononi, A. "PDM-iRZ-QPSK vs. PS-QPSK at 100 Gbit/s over dispersion-managed links", Opt. Express 20, 7895-7900 (2012).

[25]Batshon, H. G., Djordjevic, I., Xu, L., and Wang, T. "Modified hybrid subcarrier/amplitude/ phase/polarization LDPC-coded modulation for $400 \mathrm{~Gb} / \mathrm{s}$ optical transmission and beyond", Opt. Express 18, 14108-14113 (2010).

[26] Mou, C., Arif, R., Rozhin, A., and Turitsyn, S. "Passively harmonic mode locked erbium doped fiber soliton laser with carbon nanotubes based saturable absorber," Opt. Mat. Express 2, 884--890 (2012).

[27] Akhmediev, N., and Soto-Crespo , J. M. "Dynamics of solitonlike pulse propagation in birefringent optical fibers", Phys. Rev. E. 49, 5742-5754 (1994).

[28] Komarov, A., Komarov, K., Meshcheriakov, D., Amrani, F., and Sanchez, F.. "Polarization dynamics in nonlinear anisotropic fibers", Phys. Rev. A 82, 013813 1-14 (2010).

[29] Sergeyev, S. V, "Spontaneous Light Polarization Symmetry Breaking for an anisotropic ring cavity dye laser, Phys. Rev. A 59, 3909- 3917 (1999).

*tatiana.gabruseva@gmail.com; t.habruseva@aston.ac.uk. 